Abstract 719 Table 1 Distribution of HPV genotypes in cervical cancer patients

\begin{tabular}{lll}
\hline Genotype/s & No of cases & Percentage \\
\hline 16 & 69 & 69.0 \\
18 & 06 & 6.0 \\
45 & 03 & 3.0 \\
16,18 & 08 & 8.0 \\
16,62 & 02 & 2.0 \\
16,56 & 02 & 2.0 \\
16,58 & 01 & 1.0 \\
18,45 & 01 & 1.0 \\
45,39 & 02 & 2.0 \\
$16,45,56$ & 02 & 2.0 \\
$16,18,45,66$ & 01 & 1.0 \\
$16,18,53,73$ & 02 & 2.0 \\
$16,31,56,58$ & 01 & 1.0 \\
Total & 100 & 100 \\
\hline
\end{tabular}

only in multiple infections. Genotype 16 and 18 alone and as co-infection were detected in $84 \%$ cases. Among the multiple infections the most common was by HR-HR HPV types $(77.27 \%)$, whereas infections by HR-IR and HR-LR HPV types were identified in $13.64 \%$ and $9.09 \%$, respectively. Dual, triple and quadruple infection were observed in this study. Overall prevalence of HPV dual infection (16\%) was more in multiple infection. Genotypes 16/18 co-infection was more among the dual infection. Among the triple and quadruple infection 16/45/56, 16/18/53/73, 16/18/45/ 66 , and $16 / 31 / 56 / 58$ were prevalent.

Conclusion* Thus this study concluded that HPV multiple infection (22\%) is prevalent along with the single HPV infection among the cervical cancer patients from Bangladesh. Dual, triple and quadruple infection were observed in this study. Genotype 16 and 18 alone and as co-infection were detected in $84 \%$ cases. Thus the vaccination with Cervarix against HPV 16 and HPV 18 can prevent 84\% of cervical cancer in Bangladesh.

\section{MÉDECINS SANS FRONTIÈRES CERVICAL CANCER (CC) PROJECT IN MALAWI: RESULTS OF A NEOADJUVANT CHEMOTHERAPY (NACT) STRATEGY FOR LOCALLY ADVANCED CC}

${ }^{1} \mathrm{G}$ Chilinda*, ${ }^{1} \mathrm{~F}$ Husein, ${ }^{2} \mathrm{~L}$ Massamba, ${ }^{3} \mathrm{~S}$ Meja, ${ }^{3} \mathrm{~L}$ Gadama, ${ }^{3} \mathrm{G}$ Gadama, ${ }^{1} \mathrm{G}$ Sylvie, ${ }^{1} \mathrm{~N}$ Danielyan, ${ }^{1} \mathrm{~N}$ Curry, ${ }^{1} \mathrm{OP}$ Bah, ${ }^{4} \mathrm{C}$ Mambula, ${ }^{4} \mathrm{C}$ Rieux, ${ }^{4} \mathrm{C}$ Ngo, ${ }^{5} \mathrm{PF}$ Dupre. ${ }^{1} \mathrm{QECH}$ Blantyre, MSF Cervical Cancer Project, Blantyre, Malawi; ${ }^{2}$ QECH, Oncology, Blantyre, Malawi; ${ }^{3} Q E C H$, Gynaecology and Obstetrics, Blantyre, Malawi; ${ }^{4}$ Médecins Sans Frontières, Paris, France; ${ }^{5} \mathrm{CHRU}$ Brest, Breast and Gynecological Oncolgy unit, Brest, France

\subsection{6/ijgc-2021-ESGO.61}

Introduction/Background* Malawi ranks as the country with the second highest CC incidence (72.9/100 000) and mortality (54.5/100 000).

The facilities face serious challenges as the country has no functioning radiotherapy center, which leaves patients with Locally Advance Cervical Cancer (LACC) without curative options. We adopted NACT as a down-staging strategy for patients with LACC, to provide them curative options.

The aim of the survey was to assess whether NACT is an effective strategy in being able to provide adequate surgery for stage IB3, IIA2, and IIB patients.
Methodology Cohort analysis of data from women $(>=18 \mathrm{y}$. o.) receiving a Radical Hysterectomy $(\mathrm{RH})$ in MSF Malawian cancer program between December 2019 and March 2021.

Initial and post-pathology FIGO staging were described and compared according to individual characteristics and therapy received.

Comparisons of proportions were performed using the appropriate statistical test.

All analyses were performed using Stata16 (Stata Corp).

Data was extracted from Redcap Malawi and a Microsoft Excel database.

Data analyses were performed using Excel and Stata 16 (version 16.1).

Result(s)* Between December 1, 2019 and March 30, 2021, 97 women undergo RH at MSF Cervical Cancer project at Queen Elizabeth Central Hospital (QECH).

Out of these patients, 47 received NACT.

Among those patients, we observe a change in FIGO stage for 46 ( $\mathrm{p}$-value $<0.005$ ).

NACT improved post-pathology FIGO stage for 42 patients (p-value<0.005): 17 patients had complete pathological response, and 25 presented with partial response.

Four patients clinically worsened FIGO stage, requiring chemo radiation as treatment.

There was a significant decrease in histopathology results for parametrium, LVSI, LN involvement, and margin involvement (p-value $<0.005)$.

According to Clavien-Dindo Classification 12 patients had post operative complications: 5 Grade I, 1 Grade II, 4 Grade III a, and 2 Grade III b. Two patients died after NACT and $\mathrm{RH}$ died (4.3\%).

Patients that received NACT in the MSF cohort had 17.3\% less complications than non-NACT patients.

Conclusion* In the absence of radiation therapy option, NACT seems to be an effective strategy in improving patient FIGO stage enough for adequate surgery.

Long term follow up is required to assess its impact on disease free survival.

\section{CAN A CONE BIOPSY PREDICT NODAL STATUS IN EARLY CERVICAL CANCER ?}

A Berasaluce, N Martín-Calvo, E Chacon*, F Boria, N Manzour, D Vazquez, T Castellano, JÁ Mínguez, JL Alcazar, LM Chiva*. University of Navarra

\subsection{6/ijgc-2021-ESG0.62}

Introduction/Background* We aimed to identify characteristics of a cone biopsy that may independently predict higher odds of positive nodes.

Methodology We used data from the SUCCOR study, a European multicentre study that collected retrospective information of 1272 women who underwent a radical hysterectomy by open or minimally invasive surgery for stage IB1 cervical cancer (FIGO 2009) between January 2013 and December 2014. We restricted the analyses to 423 who underwent a cone biopsy. Missing values were imputed with the median in quantitative variable and grouped in a new category in qualitative ones. Univariate analysis was carried out to identify those variables related to the cone biopsy that were significantly related to nodal status. We used Student's t test for quantitative variables and Pearson's chi squared test for qualitative ones. Variables with a p value below 0.05 in the univariate analyses were introduced in a multivariable adjusted logistic regression. 


\begin{tabular}{|c|c|c|c|}
\hline \multirow[t]{2}{*}{ Variable name } & \multicolumn{2}{|c|}{ Node status } & \multirow[t]{2}{*}{ p-value } \\
\hline & Positive nodes & Negative nodes & \\
\hline \multicolumn{4}{|l|}{ Characteristics of the women } \\
\hline Age & $44.75(8.95)$ & $45.33(10.34)$ & 0.731 \\
\hline BMI & $24.92(4.85)$ & $24.71(4.35)$ & 0.816 \\
\hline Performance status & & & 0.037 \\
\hline ECOG 0 & $28(87.5)$ & $355(90.8)$ & \\
\hline ECOG 1 & $3(9.4)$ & $10(2.6)$ & \\
\hline Not reported & $1(3.1)$ & $26(6.6)$ & \\
\hline Smoker & & & 0.266 \\
\hline Yes & $24(75.0)$ & $228(58.3)$ & \\
\hline No & $4(12.5)$ & $70(17.9)$ & \\
\hline Not reported & $4(12.5)$ & $93(23.8)$ & \\
\hline \multicolumn{4}{|l|}{ Characteristics of the tumor } \\
\hline Volume of the tumor & $3427.28(6904.14)$ & $1747.19(5681.87)$ & 0.189 \\
\hline Clinical size of the tumor & & & 0.343 \\
\hline$<30 \mathrm{~mm}$ & $22(68.8)$ & $306(78.3)$ & \\
\hline$\geq 30 \mathrm{~mm}$ & $8(25.0)$ & $74(18.9)$ & \\
\hline Not reported & $2(6.2)$ & $11(2.8)$ & \\
\hline Diameter of the tumor & & & 0.006 \\
\hline$\leq 20 \mathrm{~mm}$ & $21(65.6)$ & $329(84.1)$ & \\
\hline$>20 \mathrm{~mm}$ & $11(34.4)$ & $60(15.3)$ & \\
\hline Not reported & $0(0.0)$ & $2(0.5)$ & \\
\hline Histology of the tumor & & & 0.555 \\
\hline Squamous carcinoma & $1(3.1)$ & $18(4.6)$ & \\
\hline Adenocarcinoma & $23(71.9)$ & $244(62.4)$ & \\
\hline Adenosquamous carcinoma & $5(15.6)$ & $101(25.8)$ & \\
\hline \multicolumn{4}{|l|}{ Related to the conization proceedure } \\
\hline Tumor volume & $1489.49(2188.48)$ & $1378.20(3898.83)$ & 0.799 \\
\hline Dimension of tumor in cone & $15.68(6.82)$ & $14.49(6.87)$ & 0.347 \\
\hline MRI done before cone & & & 0.990 \\
\hline Yes & $23(71.9)$ & $288(73.7)$ & \\
\hline No & $5(15.6)$ & $63(16.1)$ & \\
\hline Not reported & $4(12.5)$ & $40(10.2)$ & \\
\hline Type of cone technique & & & 0.345 \\
\hline Cold knife & $3(9.4)$ & $73(18.7)$ & \\
\hline Laser & $1(3.1)$ & $21(5.4)$ & \\
\hline LEEP or LLETZ & $25(78.1)$ & $268(68.5)$ & \\
\hline Margins in the cone & & & 0.033 \\
\hline Not affected & $2(6.2)$ & $87(22.3)$ & \\
\hline Uncertain & $0(0.0)$ & $14(3.6)$ & \\
\hline Afected & $28(87.5)$ & $253(64.7)$ & \\
\hline Linfovascular invasion in the cone & & & 0.681 \\
\hline Yes & $8(25.0)$ & $90(23.0)$ & \\
\hline No & $5(15.6)$ & $44(11.3)$ & \\
\hline Not reported & $19(59.4)$ & $257(65.7)$ & \\
\hline Tumor grade of differentiation in cone & & & 0.963 \\
\hline Grade I & $7(21.9)$ & $74(18.9)$ & \\
\hline Grade II & $13(40.6)$ & $130(33.2)$ & \\
\hline Grade III & $7(21.9)$ & $80(20.5)$ & \\
\hline Tumor histology in the cone & & & 0.010 \\
\hline Squamous carcinoma & $25(78.1)$ & $256(65.5)$ & \\
\hline Adenocarcinoma & $5(15.6)$ & $109(27.9)$ & \\
\hline Adenosquamous carcinoma & $2(6.2)$ & $3(0.8)$ & \\
\hline Depth of invasion the cone & & & 0.002 \\
\hline Superficially invades stroma & $1(3.1)$ & $81(20.7)$ & \\
\hline Invades $1 / 3$ of stroma & $2(6.2)$ & $51(13.0)$ & \\
\hline Invades $2 / 3$ of stroma & $13(40.6)$ & $76(19.4)$ & \\
\hline Endocervical curettage carried out & & & 0.471 \\
\hline Yes & $24(75.0)$ & $249(63.7)$ & \\
\hline No & $7(21.9)$ & $100(25.6)$ & \\
\hline
\end{tabular}

\begin{tabular}{llll}
$\begin{array}{lll}\text { Not reported } \\
\text { Positive endocervical curretage }\end{array}$ & $1(3.1)$ & $42(10.7)$ & 0.499 \\
Yes & $3(9.4)$ & $56(14.3)$ & \\
No & $4(12.5)$ & $44(11.3)$ & \\
$\quad$ Not reported & $25(78.1)$ & $291(74.4)$ & \\
Disease free survival & $51.69(21.64)$ & $51.52(19.47)$ & 0.967 \\
Overall survival & $51.88(21.37)$ & $52.79(18.45)$ & 0.815 \\
\hline
\end{tabular}

Result(s)* In the univariate analyses we found that large tumours $(>2 \mathrm{~cm})$ and squamous or adenosquamous histopathology were associated with higher odds of positive nodes. Regarding characteristics of the cone biopsy, we observed that deep stroma invasion (2/3 of stroma) and positive margins were also associated with higher odds of positive nodes. In the multivariable adjusted model, we observed collinearity between the characteristics of the cone biopsy and therefore they were analysed separately. When accounting for tumour size and histopathology, deep invasion of the stroma was associated with 12-fold higher odds of positive nodes, but affected or uncertain margins was not.

Conclusion* In patients after cone biopsy, the association of tumors $>2 \mathrm{~cm}$ plus deep stromal invasion $(>2 / 3)$ is correlated with the higher risk of positive lymph node in early cervical cancer.

\section{SAFETY PROFILE OF KEYTRUDA (PEMBROLIZUMAB) FOR THE TREATMENT OF PATIENTS WITH ADVANCED PD-L1 POSITIVE CERVICAL CANCER}

${ }^{1} \mathrm{~F}$ Ahmed, ${ }^{1}$ I Khan, ${ }^{2} \mathrm{Z}$ Shah*, ${ }^{1} \mathrm{R}$ Garimella, ${ }^{1} \mathrm{~S}$ Mishra, ${ }^{1} \mathrm{~A}$ Bhatnagar, ${ }^{3} \mathrm{~K}$ Andrews, ${ }^{4} \mathrm{~S}$ Zaidi, ${ }^{5} \mathrm{U}$ Salman, ${ }^{6} \mathrm{SH}$ Bobba, ${ }^{7} \mathrm{~S}$ Gudapati, ${ }^{2} \mathrm{Z}$ Yukselen, ${ }^{1} \mathrm{P}$ Guntipalli, ${ }^{8} \mathrm{~J}$ Michel, ${ }^{9} \mathrm{~S}$ Ahmad, ${ }^{10} \mathrm{R}$ Zarrar, ${ }^{11} \mathrm{G}$ Enebong Nya. 'Larkin Community Hospital, Division of Clinical and Translational Research, South Miami, USA; ${ }^{2}$ Weiss Memorial Hospital, Department of Internal Medicine, USA; ${ }^{3}$ Prince Mohammad Bin Fahad University, Department of CORE, Al Khobar, Saudi Arabia; ${ }^{4}$ Lahore Medical and Dental College, College of Medicine, Pakistan; ${ }^{5}$ Bakhtawar Amin Medical and Dental College, College of Medicine, Multan, Pakistan; ${ }^{6}$ Washington University of Health and Science, College of Medicine, USA; 'Washington University of Health and Science, College of Medicine, Belize; ${ }^{8}$ Larkin Community Hospital, Department of Gynecologic Oncology, USA; ${ }^{9}$ Advent Health Cancer Institute, Department of Gynecologic Oncology, USA; ${ }^{10}$ Mayo Clinic, Department of Oncology, Rochester, USA; ${ }^{11}$ John Hopkins Hospital, Department of Gynecologic Oncology, USA

\subsection{6/ijgc-2021-ESG0.63}

Introduction/Background* Cervical cancer is the fourth most common cause of cancer-related deaths in women worldwide. With screening for precancerous lesions and vaccination for preventive human papillomavirus (HPV), a survival improvement has been observed in these patients in developed countries. In recent years, U.S. Food and Drug Administration (FDA) approved pembrolizumab for the treatment of patients with advanced cervical cancer with disease progression on or after chemotherapy whose tumors express PD-L1. Herein, we present the first systematic review discussing the safety profile of this drug.

Methodology A systematic literature search was performed on March 2021 according to PRISMA statement using PubMed, Embase, Scopus, CINAHL, Cochrane, Google Scholar, and Clinicaltrials.gov databases without any filters. The medical search terms (MeSH) utilized to conduct the search are, ' uterine cervical neoplasms' AND 'Pembrolizumab'. After a detailed primary and secondary screening done by two members of 188 studies, only 4 studies were found that discussed the safety profile of pembrolizumab. 